ISSN 00156043

NOTAS

\title{
Aportes sobre el financiamiento del desarrollo rural con enfoque territorial en América Latina'
}

\author{
Nelson Espinoza ${ }^{2}$
}

Palabras clave: Desarrollo rural, América Latina, Inversiones, Financiamiento.

Key words: Rural development, Latin American, Investment, Financing.

\section{I.Visión general de la preinversión e inversión agrícola-rural en América Latina y el Caribe}

La agricultura y el medio rural de América Latina y el Caribe (ALC) se han caracterizado por procesos poco rigurosos de preinversión, falta de inversiones y escaso acceso al financiamiento, tanto público como privado ${ }^{3}$. En muchos de los casos no se trata de la cantidad de los recursos destinados al medio rural sino

\footnotetext{
1 Versión revisada (octubre 2007) del documento preparado para el seminario sobre desarrollo rural con enfoque territorial, organizado por la Fundación ETEA, celebrado el 27 de junio del 2007 en Córdoba, España.

${ }^{2}$ Coordinador de la Unidad de Proyectos de Inversión. Dirección de Financiamiento Externo y Proyectos de Inversión. Instituto Interamericano de Cooperación para la Agricultura (IICA).

${ }^{3}$ Preinversión: puede ser concebida como una lucha contra la incertidumbre en la asignación de recursos y normalmente incluye los estudios de proyectos que se realizan previamente a la realización de las inversiones y que sirven de base para tomar las decisiones sobre las mismas (estudios a nivel de idea, perfil, prefactibilidad y factibilidad). Inversión: es la etapa en que se realizan físicamente las inversiones, tales como obras civiles, equipamiento, otros bienes conexos y actividades que contribuyen a desarrollar las capacidades de los actores relacionados con la ejecución de la inversión.
} 
de la rentabilidad de los recursos invertidos, ya que la duplicidad de inversiones, la mala escogencia de las prioridades, las pocas capacidades de los implementadores y la inexistencia de política de acompañamiento, no permiten el óptimo aprovechamiento de los recursos ni de los activos de los beneficiarios.

Otras causas que influyen en la baja atracción de inversiones, son: (i) falta de infraestructura rural para la generación de negocios; (ii) la prestación de servicios financieros privados en el medio rural no ha experimentado el mismo crecimiento que en otros países en desarrollo (principalmente Asia), debido a que generalmente se concibe la producción agrícola como una actividad altamente riesgosa, con probabilidades de sufrir daños ocasionados por los desastres naturales, la incertidumbre en los ingresos de los productores a consecuencia de la volatilidad de los precios agrícolas y la imposibilidad de contar con garantías reales debido a la inseguridad en la tenencia de la tierra; (iii) poca articulación en las cadenas agroproductivas comerciales, que ha dejado por fuera a gran cantidad de productores primarios.

\section{I. Fuentes para la preinversión, inversión y financiamiento}

\section{I.I.I. Gasto Público}

En la mayoría de los países de ALC se esta reduciendo el gasto público en la agricultura con respecto al total del gasto (con algunas excepciones como Bolivia, Chile, Colombia y México), este patrón puede deberse a varias razones, entre ellas la tendencia en la reducción de la participación del sector primario agropecuario en las economías de la región, que se genera al incrementarse el ingreso per capita; y un bajo poder de negociación de la agricultura respecto a los demás sectores. No obstante, la participación del estado en el sector agrícola es muy variable en la región, medido en términos de gasto público como porcentaje del Producto Interno Bruto Agropecuario.

Estas diferencias reflejan diferentes grados de participación del sector público en las actividades del sector agropecuario. Por ejemplo, países con baja participación del sector primario agropecuario en la economía y con alta participación del Estado en la agricultura (gasto público en agricultura superior al $17 \%$ del Producto Interno Bruto Agrícola-PIBA) incluyen a Chile, México, Panamá, República Dominicana y Uruguay. En contraste países con más alta participación de la agricultura en la economía y con relativa baja participación del Estado en la agricultura (gasto 
público en agricultura menor al 6,5\%) incluyen a Bolivia, Colombia, Costa Rica y Paraguay (ver cuadro 1). Por otra parte, en el nivel de subregiones, la menor participación del estado en las actividades agrícolas se efectúa en los países andinos, mientras que en la región mesoamericana, con excepción de Costa Rica, el Estado participa más activamente en las actividades agrícolas que en las demás subregiones.

\section{I.I.2. La Banca Nacional de Desarrollo}

En ALC se pueden distinguir tres fases en el financiamiento del sector. Los años setenta estuvieron caracterizados por una fuerte participación estatal a través de la Banca Nacional de Desarrollo. En particular, financiando a productores no cubiertos por la banca comercial con tasas de interés preferenciales. A finales de los ochenta, como parte de los cambios estructurales llevados a cabo en las economías de la región, se adoptaron medidas para reducir la carga fiscal impuesta por créditos subsidiados, mediante liberación de los mercados financieros rurales. La banca estatal disminuyó su participación como banca de primer piso y redujo o eliminó el crédito con tasas de interés subsidiadas ${ }^{4}$.

Sin embargo, la disminución o eliminación de la oferta de servicios financieros no implicó que la demanda por crédito fuera cubierta por la banca comercial, en particular la de los productores marginales. La banca privada más bien orientó sus recursos para atender a grandes y medianos agricultores que tienen posibilidades de ofrecer garantía, si se presentan circunstancias adversas en la producción o en el mercadeo. A finales de los noventa, los bancos del estado incrementan nuevamente su participación como banca de primer piso para fortalecer la oferta de servicios financieros y se dirige con mayor precisión a productores e intermediarios financieros específicos, tomando en cuenta la participación de la banca comercial y proporcionando servicios orientados a fortalecer la demanda de crédito.

\section{I.I.3. Las Instituciones Financieras de Desarrollo (IFD)}

A inicios de 2002, los activos totales de un conjunto de 108 Instituciones Financieras de Desarrollo (IFD) en América Latina llegaban a US $\$ 360$ mil millones con

\footnotetext{
${ }^{4}$ Banca de primer piso: Son intermediarios financieros que establecen una relación directa con los empresarios o beneficiarios que solicitan crédito para realizar sus inversiones. Banca de segundo piso: Es aquella institución que canaliza recursos financieros al mercado a través de otras instituciones financieras intermediarias.
} 
una cartera neta de US\$261 mil millones canalizados al desarrollo productivo. Las IFD de América Latina son mayoritariamente entidades de propiedad pública ${ }^{5}$ y conceden un porcentaje importante de los préstamos del total otorgado por el sistema financiero de cada país. En contraste con lo que sucedía a mediados de la década pasada donde las IFD operaban fundamentalmente como banca de segundo piso, en la actualidad una mayor proporción de ellas está operando en el primer piso y en menor medida en el segundo. Algunas de las de primer piso canalizan pequeños préstamos a través de intermediarios financieros no bancarios, tales como cajas rurales y cooperativas. Ejemplos de IFD en la región incluyen: (i) Argentina: Banco de Inversión y Comercio Exterior; (ii) Brasil: Banco Nacional de Desenvolvimento Económico e Social; (iii) Chile: Banco de Desarrollo; (iv) Costa Rica: Banco Nacional de Costa Rica; y (v) México: Fideicomisos instituidos en relación con la agricultura.

Las IFD tienen una orientación preponderantemente multisectorial. Sin embargo, en el nivel sectorial los sectores: agropecuario, desarrollo rural y agroindustrial, son en conjunto, los principales receptores de financiamiento. Los componentes de una muestra de 46 IFD pertenecientes a 14 países de la región, otorgaron conjuntamente el $22 \%$ de los préstamos totales desembolsados en el 2001, seguidos por el sector vivienda (20\%) y la industria manufacturera (13\%). Las IFD no se limitan al financiamiento a través de préstamos, progresivamente están incorporando un enfoque integral para reducir el riesgo y otorgar financiamiento. Entre sus funciones operativas está cobrando importancia la asistencia técnica y capacitación; provisión de garantías y administración de fideicomisos. Adicionalmente para reducir riesgos, las IFD están siguiendo un enfoque multisectorial, por ejemplo las IFD ya no se están concentrando solamente en financiar al sector agropecuario, sino también actividades como: la vivienda rural, el desarrollo de la Pequeña y Mediana Empresa (PYME) rural y los gobiernos locales.

\section{I.4. La Banca Multilateral}

Los principales organismos internacionales para el financiamiento del desarrollo agrícola de la región están constituidas por el Fondo Internacional de Desarrollo Agrícola (FIDA), el Banco Mundial y el Banco Interamericano de Desarrollo (BID). El FIDA se concentra en el financiamiento de proyectos de desarrollo rural y alivio de pobreza y se centra en la diversificación de las actividades generadoras de

\footnotetext{
${ }^{5}$ En el año 2002 , alrededor del $68 \%$ de ellas era de carácter público, y representaban el $94 \%$ de los activos de la banca de desarrollo (ALIDE, 2004).
} 
ingresos de las familias y en el establecimiento de vínculos con actividades de valor agregado, como la transformación de productos agrícolas en pequeña escala y la apertura de mercados fuera de la comunidad beneficiaria 6 . Para finales de 2005 , la cartera regional del FIDA comprendía 30 programas y proyectos para $\mathrm{ALC}$, lo cual era equivalente a una inversión total de US $\$ 460,7$ millones. Dentro de la cartera de préstamos regionales, es importante señalar que ha aumentado el número de países que reciben préstamos en condiciones ordinarias; además, las donaciones continuaron desempeñando una función importante dentro de la estrategia del FIDA en la región.

Durante 2004, el Banco Mundial otorgó financiamiento al sector agropecuario, forestal y pesquero por US $\$ 379,5$ millones ( $7 \%$ del total de préstamos a ALC). El banco ha enfocado fuertemente su acción al combate de la pobreza. En el medio rural ha potenciado los temas sobre manejo sostenible del ambiente y recursos naturales; para apoyar el desarrollo rural impulsa iniciativas en servicios financieros y administración de tierras. En estos temas, el banco financió préstamos en la región por un total de US\$408,7 millones en 2004 , cifra que bajó a $\$ 236,5$ millones en 2006.

El BID en el período de 30 años transcurridos desde 1961 a 1990, manejó una cartera para la agricultura que representaba el $20 \%$ del total de préstamos. En la década de los noventa, la cartera de la agricultura representó apenas el $4 \%$ en promedio, o sea que el sector agropecuario productivo dejó de ser prioritario en la perspectiva del desarrollo económico social del BID para la región. Esta situación financiera cambio en el año 2001, cuando el Banco asignó US\$683,2 millones en préstamos para el sector, pero nuevamente para el año 2002, se redujeron los préstamos a la agricultura. La cartera agropecuaria durante ese año fue de US\$ 156,9 millones que representa el 3,4\% del total de préstamos.

Durante 2006, la cartera de los proyectos del BID en ejecución aumentó en $\$ 1.066$ con la aprobación de 36 nuevos préstamos rurales o con algún componente de

\footnotetext{
${ }^{6}$ De una muestra de 15 proyectos en 7 países se pudo determinar que el 59 por ciento del monto financiado por el Fondo se orienta hacia proyectos de "desarrollo rural" en los cuales se incluyen actividades productivas agrícolas y no agrícolas, en sus diferentes fases del proceso productivo (primario y en algunos casos secundario), comercialización de la producción, así como infraestructura de apoyo a las comunidades y la producción. Un 26 por ciento del monto total ha financiado proyectos de "desarrollo agrícola" que comprenden actividades que se orientan hacia la producción y comercialización de los productos agrícolas. Casi el 11 por ciento del monto financiado es orientado a proyectos que están desarrollando mecanismos flexibles para el otorgamiento de crédito a productores y sus organizaciones para actividades productivas en el área rural.
} 
inversión en ese sector. Los nuevos préstamos otorgados incluyen tanto proyectos dirigidos al sector rural-agropecuario como proyectos que benefician a áreas rurales y a su población. En términos generales, el $49 \%$ de estos 36 nuevos préstamos aprobados se destinaron a inversiones en el sector de transportes y telecomunicaciones, principalmente en proyectos de mejora, rehabilitación y creación de infraestructuras. Del restante, el $20 \%$ fue para el desarrollo rural (proyectos de desarrollo sostenible socioeconómico de comunidades rurales, creación de agencias de desarrollo y mejora de destinos turísticos), el 9\% para salud y educación, el $7 \%$ para misceláneos, el $5 \%$ para tecnología, el 3\% para agropecuario y electricidad, el $2 \%$ para comercialización y el $1 \%$ para agua, saneamiento y tierras.

\section{I.2. Breve análisis de perspectivas sobre la preinversión, inversión y financia- miento agrícola-rural}

Los países están promoviendo acciones en dos temas multisectoriales fundamentales para su desarrollo: (i) alivio de la pobreza: impulsando estrategias de lucha contra la pobreza (ELP) en el marco de los países pobres altamente endeudados (HIPC), requisito básico para tener acceso a recursos de cooperación técnica y financiera orientados a lograr los Objetivos de Desarrollo del Milenio; y (ii) mejoramiento de la competitividad: para enfrentar los retos y aprovechar las oportunidades de la globalización y la apertura comercial, para lo cual impulsan el mejoramiento de las instituciones, los sistemas de gestión, los mecanismos de transparencia, el capital humano, la infraestructura, la innovación, etc. Esta acción se enmarca, en especial, en las llamadas Estrategias de Acompañamiento o Paralelas a los procesos de negociación e ejecución de tratados comerciales, en los que, por lo general, son los Ministerios de Finanzas y Economía los que lideran las acciones junto con los de Relaciones Exteriores y de Comercio, para respaldar la apertura comercial.

Las iniciativas de inversión sectorial agropecuaria con proyectos temáticos específicos son cada vez más escasas (tecnología y extensión, riego, drenaje, salud animal, sanidad vegetal, desarrollo ganadero, tierras, comercialización agropecuaria, otros). La tendencia es que ahora se han redefinido y actualizado los temas en el marco de la agricultura ampliada y las cadenas agroproductivas comerciales; $y$ cada vez, con mayor frecuencia, forman parte de las inversiones públicas multisectoriales; como ejemplo, se citan los siguientes: sanidad agropecuaria e inocuidad de alimentos; "trazabilidad"; gestión integrada de recursos hídricos; innovación tecnológica; negociaciones comerciales; administración de tierras; reconversión agroempresarial; agronegocios; economía rural, otros. 
La tendencia es que las iniciativas de inversión pública sectorial, especialmente cuando hay cofinanciamiento de bancos multilaterales, se incluyen como actividades dentro de componentes y subcomponentes en nuevas modalidades de productos financieros multisectoriales de reforma de política que impulsan los Gobiernos, dirigidos a mejorar la competitividad y al iviar la pobreza; por lo tanto, los Ministerios o Secretarías de Agricultura cada vez tienen menor protagonismo en la negociación y ejecución de inversiones públicas, y son los Ministerios de Finanzas, Economía, Planificación, Relaciones Exteriores y Comercio los que negocian y ejecutan.

Existe una clara tendencia, que se inició durante la década pasada, sobre el aumento sustantivo de la inversión pública en programas y proyectos de recursos naturales y ambiente, con productos financieros blandos, no reembolsables y donaciones. Como componentes, subcomponentes de esos programas y proyectos que apoyan a pequeños productores, comunidades rurales y pueblos indígenas, se incluyen, por ejemplo: producción agrícola sostenible; agricultura orgánica; agricultura étnica; agrobiodiversidad; ecomercados; servicios ambientales; agroturismo; turismo rural y otros.

En síntesis, la preinversión, inversión y financiamiento agrícola-rural se esta dirigiendo a dar respuesta a los requerimientos para mejorar la competitividad y demandas de los mercados internacionales, tanto en desarrollo de capacidades institucionales y de los recursos humanos, como en infraestructura y equipo, en áreas tales como: (i) sanidad agropecuaria e inocuidad de alimentos ("trazabilidad"; aumento de la capacidad diagnóstica y modernización de laboratorios; fortalecimiento de los sistemas de prevención de ingreso y vigilancia epidemiológica; control y erradicación); (ii) innovación tecnológica ("monitoreo" y capturas tecnológicas, difusión); (iii) gestión integrada de recursos hídricos (gestión del riego, riego y drenaje, aguas superficiales y subterráneas, manejo sostenible de cuencas hidrográficas); (iv) desarrollo productivo en procesos de administración de tierras para titulación y registro de la propiedad; $(v)$ negociaciones comerciales y agronegocios (normas, mecanismos e instrumentos para su promoción y desarrollo); (vi) mecanismos e instrumentos financieros innovadores como los fondos competitivos. 


\section{Hacia la asignacion de recursos para el desarrollo rural con enfoque territorial: la propuesta del Instituto Interamericano de Cooperación para la Agricultura ${ }^{7}$}

La persistencia de altos niveles de pobreza de ALC y la poca efectividad de los programas y proyectos de combate de la pobreza, y, en particular, la pobreza rural, hacen necesario mejorar el diseño y ejecución de estrategias e instrumentos (medidas de política, programas y proyectos de inversión), e incorporar nuevos enfoques y métodos. En este sentido, se están promoviendo y ejecutando en ALC, con mayor frecuencia e intensidad, iniciativas de desarrollo rural con enfoque territorial, entendido como un proceso de transformación productiva e institucional, interpretando lo rural en su dimensión territorial, multisectorial, incluyendo actividades y relaciones económicas, medio-ambientales y sociales.

La propuesta conceptual, metodológica y operativa del Instituto Interamericano de Cooperación para la Agricultura (IICA), sobre el desarrollo rural con enfoque territorial, apunta a aumentar la contribución de la agricultura y del medio rural a la generación de empleo y a la reducción de la pobreza rural, sustentado en los conceptos de: (i) Cadenas agro-productivo-comerciales: definidas como una visión ampliada de la agricultura, a partir de sus encadenamientos con otros sectores de la economía; y (ii) Territorios rurales: que constituyen una visión integradora de la economía rural, en la que destacan, además de la agricultura: la economía rural no agrícola; los vínculos entre la economía rural no agrícola y la agricultura; y las funciones múltiples del binomio territorio/agricultura. Junto con estos dos conceptos, la propuesta incluye acciones para materializar el enfoque mediante: (i) Innovación institucional; y (ii) Desarrollo de capacidades estratégicas para el desarrollo rural. Asimismo, la propuesta establece que la heterogeneidad es un signo distintivo de la agricultura y el medio rural; esto incluye: diversidad de agriculturas entre países, la variedad de agriculturas, productores agrícolas, cadenas agro-productivo-comerciales y territorios rurales en el interior de los países; y la diversidad de la base de recursos naturales y de actores sociales en el interior de los territorios rurales.

\footnotetext{
7 Esto corresponde a la política corporativa oficial del IICA respecto al tema de desarrollo rural con enfoque territorial contenida en el documento: IICA (septiembre 2006), "Políticas para la Prosperidad Rural. Informe especial sobre la contribución de la agricultura y de los territorios rurales a la reducción de la pobreza, la creación de empleo y la promoción de la prosperidad rural". También contenida en la Serie de documentos: Sinopsis del Instituto. Desarrollo Rural Sostenible N5- 2005. Todo el contenido de este capítulo procede total o parcialmente de dichos documentos.
} 


\section{I. Cadenas agroproductivo comerciales y los agronegocios}

Las cadenas concatenan procesos y relaciones de producción y distribución, que entrelazan lo rural con lo urbano, con diferentes actores económicos y sociales, desde la producción hasta la entrega del producto al consumidor final. Una porción importante de la prosperidad rural se puede generar mediante una inserción de los pequeños productores en las cadenas agro-productivo-comerciales y los agronegocios que potencie las sinergias con otros sectores de la economía. Para maximizar el impacto de las cadenas agro-productivo-comerciales y de los agronegocios en la generación de empleo y la reducción de la pobreza, el IICA propone:

i. Fomentar una gobernabilidad incluyente de las cadenas: la gobernabilidad incluyente debe facilitar la inserción de los 'socios' más débiles y promover el desarrollo de mecanismos de negociación y de acciones que minimicen las asimetrías de poder.

ii. Potenciar la legislación de sanidad y las buenas prácticas agrícolas: las nuevas normas que surgen de la apertura comercial, incluida la determinación de reglas ambientales y sociales, ofrecen oportunidades para generar empleo e ingresos rurales adicionales.

iii. Promover la innovación para la inserción productiva de los agricultores pobres: se deben crear condiciones para transformar procesos de desarrollo tecnológico en procesos de innovación tecnológica, y fortalecer la articulación entre los esfuerzos de investigación y las demandas/necesidades de los campesinos.

iv. Complementar la agenda de apertura para potenciar el papel del comercio en la reducción de la pobreza rural. Las negociaciones comerciales son importantes: sin embargo, la verdadera negociación se hace en la agenda interna, la cual debe responder a las necesidades de reestructuración y modernización de la producción nacional, para enfrentar las amenazas y oportunidades del nuevo escenario comercial.

v. Desconcentrar la tenencia y fortalecer el acceso de la población rural a la tierra: las políticas de tierras deben adoptar formas flexibles de acuerdo con las particularidades locales y no un modelo único. Se requiere la implementación de políticas y el manejo de una multiplicidad de instrumentos que se combinarían según las especificidades de los países, regiones y territorios.

vi. Una nueva visión para las inversiones: los lineamientos esbozados en la propuesta 
hacen necesaria una visión diferente sobre los criterios para la asignación de los recursos de inversión. Se deben impulsar proyectos que fomenten las sinergias entre las cadenas agro-productivo-comerciales y los territorios, que promuevan encadenamientos de la agricultura con otros sectores, que contribuyan a crear y estimular economías de escala y de aglomeración, que potencien el desarrollo de nuevas actividades productivas, que impulsen la asociación y concurran a potenciar y movilizar el ahorro que se genera en el interior de los territorios y que coadyuven a fortalecer el capital humano y social de los territorios rurales. Como mecanismos e instrumentos probados para materializar este tipo de iniciativas se incluyen los fondos competitivos. Además, es necesario dar espacio a la población rural para que participe en las decisiones sobre estrategias y prioridades de inversión. Finalmente, se requiere ampliar el rango de opciones públicas y privadas para el financiamiento de inversiones agrícolas y de desarrollo rural. Entre las opciones innovadoras a explorar están los recursos que puedan generar los mercados de servicios ambientales (e.g. el mecanismo de desarrollo limpio del Protocolo de Kyoto, como opción para el financiamiento de inversiones en biocombustibles) y, en algunos países, los recursos liberados como resultado de la condonación de deudas (e.g. Honduras, Nicaragua, Bolivia y Guyana).

\section{Recuadro 1: Recomendaciones para el financiamiento de las cadenas agroproductivo comerciales}

El desarrollo rural debe buscar cómo insertar al agricultor en cadenas de valor. Este debe ser el objetivo, porque si no está insertado, va a quedar aislado, en una situación complicada y pobre. Mirando hacia el futuro, hay que seguir invirtiendo en la organización, en la coordinación y en el mejoramiento de los productos y los procesos. En un mundo globalizado, se tiene que producir para un consumidor que cada día es más exigente en términos de calidad: (i) el gran reto es, entonces, organizar a los productores para producir para un mercado de calidad y en el menor tiempo posible; (ii) se debe invertir mucho más en el manejo de riesgo; hay que darles más instrumentos a los intermediarios financieros para que ellos se puedan acercar a este campo y empezar a financiar a las cadenas; (iii) tenemos que invertir en préstamos más estructurados que atiendan todos los diferentes intereses y con capacidad de asumir el riesgo a lo largo de la cadena; y (iv) hay que pensar más en nuevos tipos de fondos o líneas de crédito, para prestar a mediano y largo plazo y para aumentar la capacidad de movilizar depósitos domésticos. El financiamiento en agricultura es muy diferente al de microfinanzas, por ejemplo. Para tener impacto, para tener éxito, hay que prestar a plazos de dos, cinco, siete años. 
El financiamiento de las cadenas agrícolas de valor no consiste en prestar por un año; para tener agronegocios exitosos y fuertes, debe prestarse a plazos más largos.

Fuente: Academia de Centroamérica; FAO; RUTA; Serfirural: Financiamiento de las Cadenas Agrícolas de Valor. 2007

\subsection{Territorios rurales}

Los territorios rurales son ámbitos geográficos con una historia propia y una base común de recursos naturales, donde ocurre la actividad agrícola y se dan relaciones socio-culturales, técnico-económicas y político-institucionales que vinculan a los actores del agro entre sí, con su ambiente natural, con otras actividades no agrícolas y con núcleos urbanos. Existen lecciones aprendidas que permiten obtener valiosas enseñanzas para mejorar las intervenciones en los territorios rurales, para ello se presentan dos casos: desarrollo fronterizo Costa Rica-Nicaragua (Anexo 1), y el Nordeste en Brasil (Anexo 2). Para potenciar el papel de los territorios rurales en la generación de empleo y la reducción de la pobreza, la propuesta del IICA propone:

i. Vincular la economía agrícola y la economía rural no agrícola. Estos vínculos tienen un doble sentido de integración: en el interior de los territorios rurales son elementos que dinamizan las economías locales; y en su nivel de articulación regional y nacional, imprimen mayor dinámica a las áreas rurales.

ii. Promover la competitividad de los territorios: se debe fortalecer la competitividad sistémica de los territorios (i.e. las condiciones que estos les ofrecen a las empresas que se asientan en ellos), la cual está determinada principalmente por la provisión de bienes públicos. El objetivo es crear territorios eficientes que favorezcan empresas eficientes que retribuyen a la sociedad por la vía de mayores rentas sociales.

iii. Promover economías de aglomeración: las economías de aglomeración son una opción plausible para enfrentar las ventajas competitivas de las economías de escala de las grandes empresas, creando conjuntos de pequeñas y medianas empresas que logran generar volúmenes de producción importantes, que a la vez contribuyen a generar economías de localización y proximidad de gran potencial.

iv. Dinamizar mercados rurales de trabajo: se enfatiza la necesidad de poner mayor atención a la promoción de una nueva economía de mercados laborales, a partir del estímulo al trabajo femenino en las actividades no agrarias y la bús- 
queda de fuentes alternativas de empleo en la cultura, el ocio, los servicios y el medio ambiente.

v. Fomentar la agroindustria rural: se destaca la importancia de la agroindustria rural y de los vínculos de esta con los agronegocios para la reducción de la pobreza rural, mediante la generación de empleos y la valorización de la producción campesina.

vi. Potenciar nuevas dinámicas territoriales y actividades productivas: la propuesta destaca la necesidad de superar las formas tradicionales en que el campo, las actividades primarias y la población rural-agrícola se relacionan con las ciudades. La revaloración de las actividades agrícolas rurales, además de su función productiva y de seguridad alimentaria, debe contemplar el reconocimiento de los servicios que estas prestan, así como de otras actividades relevantes que también tienen su base en la utilización de los recursos naturales.

vii. Garantizar equidad en el acceso al agua: se subraya que el acceso al agua como recurso productivo debe ser equitativo $y$, por lo mismo, no puede estar definido únicamente en función de la capacidad de pago de los productores. Se enfatiza también que la gestión de proyectos de infraestructura de riego debe contemplar mecanismos para la participación de las comunidades que se vean afectadas o beneficiadas con esas obras, así como mecanismos para la gestión de conflictos.

viii. Potenciar activos históricos y culturales: se reconoce que el conjunto de los activos históricos y culturales de las comunidades rurales representan un patrimonio territorial valioso como factor para su reactivación y desarrollo.

\section{Recuadro 2: Cadena de valor moderna: los suplidores de la ca- dena se financian mejorando su condición de sujetos de crédito (Caso sobre Hortifruti en Centroamérica).}

Como parte del estudio de Hortifruti, fuimos a Nicaragua, fuimos a Honduras, estuvimos en Costa Rica, desde La Irma hasta Talamanca, visitando a productores que seleccionamos nosotros mismos, de una lista de suplidores de Hortifruti, a fin de verificar nuestra intuición. Planteamos primero la hipótesis de que la cadena de supermercados es una fuente de crédito. ¿ Se verificó la hipótesis o no? La respuesta es no. Los supermercados no financian a los agricultores, como política institucional. A veces lo hacen, en casos excepcionales, cuando 
ya no hay más remedio, y entonces adelantan algunos insumos, pero esto es poco usual.

En nuestra muestra no encontramos un solo productor que hubiera sido financiado por Hortifruti mismo y ¿̇por qué? Porque cada cadena estaba creciendo, porque la Corporación de Supermercados Unidos, a la cual pertenece Hortifruti, necesitaba todo el dinero posible para financiar sus nuevas tiendas, entre otros aspectos. Una cadena dinámica tiene fuertes demandas internas de liquidez. Cuanto más dinámica, menos va a disponer de liquidez excedente para financiar préstamos entre actores de la misma cadena. Además, envuelta en un proceso complejo de competencia, las ventajas comparativas de la corporación están en el mercado, no en el financiamiento.

La nueva perspectiva, la hipótesis dos, propone que los suplidores de la cadena de supermercados mejoran su condición de sujetos de crédito gracias a la existencia de las relaciones contractuales con el comprador institucional. Estas relaciones contractuales implican varias dimensiones, capaces de limitar los riesgos enfrentados por el acreedor. El riesgo de mercado se reduce con la garantía de volumen de ventas implícita en la relación con el comprador institucional. Esta relación también reduce el riesgo de precio y, por la continuidad de las ventas a la cadena de supermercados a lo largo del año, reduce los riesgos de falta de liquidez del productor. Gracias a un plan de siembra y ventas escalonadas, conforme a instrucciones del comprador institucional, el productor tiene liquidez todo el año. Con la asistencia técnica, información de mercado y otros servicios no financieros prestados por la cadena de supermercados se reducen los riesgos de productividad, los riesgos ambientales y los riesgos de rechazos por baja calidad, al mismo tiempo que se extienden los horizontes, aumenta la inversión y se promueve la innovación.

Fuente: Academia de Centroamérica; FAO; RUTA; Serfirural: Financiamiento de las Cadenas Agrícolas de Valor. 2007

\subsection{Innovación institucional}

La persistencia de la pobreza indica que el sistema político posee fallas que superan los mecanismos que el mercado provee para solucionarla, por lo que obliga a transformar el papel que compete al Estado, de manera que, además de propiciar un escenario favorable a la inversión y garantizar la creación de empleo, se comprometa a promover la inclusión de la población pobre en la sociedad y en 
los beneficios del desarrollo. La propuesta destaca la necesidad de replantear los marcos institucionales y para ello se propone:

i. Mejorar la coordinación entre instituciones y desarrollar nuevos mecanismos: es necesario mejorar no solo la coordinación de las instituciones que trabajan en un territorio o hacen parte de él, sino también proponer mecanismos que integren esfuerzos eficazmente y potencien el intercambio y el diálogo entre las diversas instituciones responsables del diseño y la ejecución de políticas.

ii. Fomentar una institucionalidad activa: se requiere una institucionalidad activa que facilite la ejecución, la coordinación y la interlocución de las redes públicas que constituyen el Estado y las redes ciudadanas que trabajan en cada territorio.

iii. Fortalecer la cooperación: se destaca la necesidad de una institucionalidad que promueva la cooperación y la convergencia entre el Estado, la empresa privada y la sociedad civil.

iv. Promover el fortalecimiento de los actores locales: la transformación institucional debe conducir a dejar atrás la óptica sectorial y promover la transferencia de poder político hacia los gobiernos locales y la sociedad civil, en materias relacionadas con la asignación de recursos y capacidades para la toma de decisiones.

\subsection{Desarrollo de capacidades estrategicas para el desarrollo rural}

La propuesta establece que se requieren nuevas actitudes, habilidades, destrezas y conocimientos de la población local para ejercer su autonomía con responsabilidad y manejar exitosamente sus emprendimientos, nuevas habilidades institucionales para forjar relaciones de colaboración, así como una nueva práctica profesional de los funcionarios públicos. La propuesta destaca la importancia de:

i. Desarrollar capacidades para aprovechar las nuevas oportunidades productivas: son muchas las oportunidades de inserción de los pobres en la economía de los territorios. Las inversiones públicas y privadas en infraestructura o en el aparato productivo, las innovaciones tecnológicas, las interacciones entre lo urbano y lo rural, los movimientos poblacionales y los cambios institucionales crean nuevas oportunidades de negocios, modifican las estructuras socioeconómicas vigentes y la importancia de los agentes y su inserción en la economía del territorio. Sin embargo, en muchos casos se observa que los pobres rurales no tienen condiciones para aprovechar las oportunidades creadas en el territorio, mucho menos 
para transformar el desarrollo potencial en realidad. Por ello, uno de los retos es capacitar a los pobres rurales en los territorios más rezagados tanto para crear oportunidades como para aprovechar las existentes de manera más efectiva; de allí que: (a) la asistencia técnica pasa a desempeñar otros roles decisivos, como transferir conocimientos de mercado, contribuir a la formación de organizaciones de campesinos, transmitir conocimientos sobre gestión de negocios rurales; (b) es necesario continuar el esfuerzo de perfeccionamiento de nuevas metodologías de capacitación compatibles con las condiciones específicas de la población rural y el tamaño de la demanda; (c) es necesario valorizar los llamados conocimientos tradicionales; (d) es clave potenciar el conocimiento como recurso estratégico para concretar los objetivos del desarrollo sostenible de la agricultura y del territorio rural en lo que respecta a la creación de empleo y a la reducción de pobreza.

ii. El mejoramiento de la educación agrícola y rural: los programas de educación agrícola y rural en el continente han venido enfrentando una serie de problemas en sus diseños curriculares, en: educación agrícola superior y media; en los programas de capacitación; y en los programas de educación básica rural. Las opciones para mejorar son: (a) la calidad en la ampliación de la cobertura; (b) la calidad en la eficacia del sistema; (c) la calidad en la gestión pedagógica; (d) la calidad en la gestión administrativa. Bajo esta perspectiva la calidad tiene una asociación con la pertinencia educativa, en cuanto se pretende responder de una manera más eficiente, cultural y administrativamente, a las necesidades educativas de la población. Se trata de mejorar los logros de los alumnos, de ofrecer un currículum significativo, de implementar las exigencias del desarrollo educativo. Sin embargo, esta dimensión es la que ofrece mayor conflictividad por cuanto las unidades territoriales requieren de un potencial suficiente y específico para alcanzar la pertinencia deseada por medio de procesos de descentralización educativa.

iii. Promover y fortalecer las organizaciones de los agricultores pobres: uno de los puntos más vulnerables de la producción campesina es su atomización, tamaño y escala de producción, no siempre adecuados para alcanzar niveles sustentables de generación de renta. Ante esta restricción "casi estructural", la organización en sus diversas modalidades aparece como el principal -y a lo mejor el único-camino para superar la atomización. Se requiere fortalecer la capacidad de gestión de las organizaciones, la formación de líderes, el ejercicio del liderazgo y la diseminación de la cultura asociativa.

iv. Equidad en el fortalecimiento de las identidades culturales: la revaloración de la cultura como principio fundamental de integración y riqueza de una sociedad, implica una reconstrucción democrática de sociedades que tradicionalmente han 
privilegiado la homogeneización cultural y la marginación de las culturas diferentes. Algunos países, como es el caso de Bolivia, Colombia y Ecuador, han incorporado de manera explícita en sus reformas constitucionales los derechos de los pueblos indígenas, lo cual se ha reflejado en políticas educativas y de representación política dirigidas a facilitar la afirmación cultural de estos pueblos. En Ecuador, Bolivia, México y Guatemala, principalmente, se observan acciones dirigidas a promover la generación de empleo e ingresos entre los indígenas, así como a mejorar el desempeño de las empresas rurales de poblaciones indígenas, dándole gran importancia a la cultura y al manejo ambientalmente amigable, como medios para incrementar el valor de la producción.

v. Responsabilidad social empresarial en las estrategias de negocio de las empresas: estos esfuerzos consisten principalmente en políticas internas de las empresas orientadas a adoptar las normas medioambientales y a mejorar las condiciones de trabajo y el bienestar laboral. Son notables los resultados derivados de la implementación de las buenas prácticas agrícolas, como el manejo integrado de plagas, la forestación de las dunas, las prácticas de conservación de la biodiversidad, el uso y manejo seguro de agroquímicos, la disposición apropiada de los residuos, la implementación de pozos sépticos y letrinas apropiadas, entre otras, para reducir los impactos ambientales negativos sobre el agua, el suelo y el aire. Estas prácticas se han implementado con mucha creatividad, optimizando el uso de los recursos, de manera que la inocuidad y la calidad no son exclusivas de las grandes empresas.

vi. Fortalecer el capital social y la solidaridad: se entiende por capital social toda acción de relacionamiento que conlleve un impacto positivo en la condición económica de los individuos. La sola acción de los actores sociales constituye su mayor activo, se nutre de la energía de las relaciones, redes y vínculos sociales, que sirven para satisfacer propósitos específicos de supervivencia, reproducción o mejoramiento de condiciones de vida. La inversión en educación y en capacitación, y el grado de aprovechamiento de éstas por parte la población condicionan el aporte de los individuos a una red, organización o institución. La potenciación de las capacidades de la población y su integración en un proceso de relaciones horizontales, amplía la competencia de las instituciones, entidades fundamentales para el desarrollo. A su vez, las redes pueden reforzar procesos de aprendizaje (incluido el autoaprendizaje) de destrezas específicas orientadas al desarrollo sostenible. Algunos casos concretos de inversión en el fortalecimiento de capital social para el desarrollo sostenible microrregional, como el del nordeste brasileño, demuestran que esta es una forma sostenible de resolver problemas y de reorientar el desarrollo rural, siempre y cuando se impulsen valores que contemplen la cultura y el ambiente. 
vii. Desarrollo de capacidades y capital humano: el despliegue productivo de capacidades de la población rural resulta indispensable para el desarrollo sostenible. En este sentido, el potencial de cada persona es el recurso fundamental para el desarrollo de un país, una región o un territorio. No obstante, para que la población rural pueda desplegar al máximo su potencial debe estar en condiciones de acceder a los bienes y servicios básicos -entre ellos la educación y la capacitación-, sin los cuales sería impensable que pudieran responder a los retos del desarrollo.

viii. Desarrollar capacidades para la cooperación local: el enfoque territorial del desarrollo rural se corresponde con una forma particular de organización de los procesos de desarrollo. En esencia, la meta es lograr la mayor eficiencia posible en la gestión de cada uno de los agentes que participan en un espacio dado, mediante la construcción de sinergias, enlaces, formas comunicativas, alianzas, solidaridades, identificación de ventajas territoriales y compromisos de aprovechamiento. Este proceso se expresa en modelos de cooperación local, donde los distintos miembros de una comunidad se asocian libremente en formas de organización autónoma para gestionar un proyecto territorial local. La cooperación local tiene tres pilares: (a) la información, el conocimiento y la creatividad en la formulación de un proyecto local; (b) la organización, el compromiso y el control en la gestión y ejecución de un proyecto propio; y (c) el empoderamiento para decidir, presionar, gestionar y actuar colectivamente. La cooperación implica la identificación de cada una de las competencias, responsabilidades y funciones de los agentes públicos y privados y crear las condiciones para que la interacción entre ellos redunde en beneficios para todos. En un modelo de cooperación, la iniciativa de los procesos de desarrollo se gesta en la relación entre los actores y no en un agente externo o exclusivamente público. Esta visión se opone a la que tradicionalmente ha caracterizado la política pública sectorial, en donde la iniciativa parte de los gobiernos nacionales.

\section{Recuadro 3: Aspectos relevantes del desarrollo rural con enfo- que territorial de Brasil}

La organización social y el capital social son elementos fundamentales para la conceptualización y delimitación de un territorio. Hoy día, por ejemplo, los habitantes del Seridó reconocen su territorio no solo por sus características climáticas, geográficas y demográficas, sino por las 450 asociaciones comunitarias, 25 cooperativas, 26 sindicatos rurales, 28 consejos municipales, 2 asociaciones de municipios y un consejo de desarrollo regional que lo con- 
forman. Además, ven en él las redes de cooperación y confianza que se han establecido entre las distintas asociaciones.

Para que tengan permanencia en el tiempo, las acciones de desarrollo local sostenible referidas a la movilización de la sociedad, a la organización de los actores sociales y a la creación de instancias deliberativas, deben implementarse en el contexto de proyectos de inversión, siguiendo el ejemplo de los proyectos de combate a la pobreza rural (PCPR).

La generación de empleo y renta considera actividades agrícolas y no agrícolas para garantizar la sostenibilidad económica del desarrollo de los territorios rurales, por medio de la diversificación de sus economías. Las cadenas de agronegocios son vistas como una estrategia de generación de empleo, renta y fortalecimiento del capital social.

La complementación de acciones macro con acciones micro es fundamental para la sostenibilidad del desarrollo. La existencia de millares de pequeños proyectos comunitarios en el Seridó no confirió la escala para la sostenibilidad del desarrollo de la región, y sirvió apenas para resolver problemas puntuales. Este esfuerzo deberá complementarse con la ejecución de programas de inversión de ámbito regional, como los programas de recursos hídricos y de cadenas de agronegocios previstos en el plan de desarrollo de la región

Fuente: IICA/CMiranda y AMatos. 2002. Brasil: Desarrollo rural sostenible con enfoque territorial: La experiencia del IICA en Brasil.

\subsection{Consideraciones finales}

La propuesta se inserta también dentro de las acciones del IICA en apoyo del proceso de transición institucional de la agricultura y del medio rural que se ha venido dando desde finales de la década de los ochenta. Un proceso que derivó en transformaciones que afectaron de diversa manera los ministerios de agricultura y de desarrollo rural, así como el papel que corresponde al sector privado y a sus organizaciones en el proceso de desarrollo y en la gestión privada de funciones públicas. La propuesta destaca la necesidad de enfrentar una serie de retos que a continuación se presentan:

i. Superar la visión sectorial del medio rural, que lo identifica como sinónimo de lo agrícola, a favor de una visión multisectorial del territorio, que 
incorpora elementos de la economía regional y de la economía de recursos naturales.

ii. Articular las dimensiones urbana y rural de manera orgánica, integrando espacios demográficos que comparten estructuras sociales, económicas e institucionales construidas en procesos históricos de apropiación del espacio, y en particular, de sus recursos naturales.

iii. Complementar los enfoques de participación y compensación con mecanismos que promuevan también la cooperación, responsabilidad compartida, cogestión e inclusión económica y social.

iv. Potenciar la visión convencional de manejo de recursos naturales desde una perspectiva de protección ambiental y de producción limpia.

v. Superar la visión de transferencia tecnológica como herramienta para incrementar la productividad, a partir de los conceptos de innovación tecnológica y competitividad territorial, enfatizando una visión de competitividad sustentada en la interacción de aspectos económicos, sociales y ambientales.

vi. Trascender la visión convencional del capital, la cual se restringe al capital económico, destacando también la importancia de la creación de capital humano (capacidades de la personas), la valorización del capital social (relaciones, redes, etc. que facilitan la gobernabilidad y amplían el acceso a servicios básicos), la conservación y uso sostenible del acervo de capital natural (base de recursos naturales), así como el fortalecimiento de las capacidades culturales y políticas de la sociedad rural.

vii. Promover esquemas de cooperación que resulten en iniciativas de desarrollo rural orientadas por las demandas de los pobladores y agentes de los territorios, como forma efectiva de enfrentar la diversidad y heterogeneidad con estrategias diferenciadas en el territorio y de complementar los esquemas convencionales de gestión de arriba hacia abajo.

viii. Promover la gestión del conocimiento, como herramienta para el descubrimiento, adquisición, sistematización, diseminación y gestión de buenas prácticas para gestionar el desarrollo de la agricultura y del medio rural.

ix. Desarrollar nuevos mecanismos de gestión, que promuevan la articulación entre lo público y lo privado y entre lo nacional, lo regional y lo local. 
$x$. Potenciar las sinergias intersectoriales y multisectoriales, con mecanismos e instrumentos innovadores, desde las agrocadenas agroproductivas comerciales y en todos los procesos de gestión de territorios rurales. El éxito requiere asimismo de políticas innovadoras y la creación de nuevas capacidades entre los hacedores de política, los empresarios, los técnicos y los líderes locales.

\begin{tabular}{|c|c|c|c|c|c|c|}
\hline \multicolumn{7}{|c|}{$\begin{array}{l}\text { CUADRO 1. Gasto público y la participación en el PIB total y Agropecuario } \\
\text { (Millones de US\$ a precios constates, 1995=100) }\end{array}$} \\
\hline & \multirow{2}{*}{\begin{tabular}{|c|c|}
$\begin{array}{c}\text { Gasto } \\
\text { público } \\
\text { Agropecuario }\end{array}$ \\
Promedio \\
1998 - 2000 \\
\end{tabular}} & \multirow{2}{*}{$\begin{array}{c}\text { PIB total } \\
\text { Promedio } \\
1998 \text { - } 2000\end{array}$} & \multirow{2}{*}{$\begin{array}{c}\text { PIB Agrop } \\
\text { Promedio } \\
1998 \text { - } 2000\end{array}$} & \multirow{2}{*}{$\begin{array}{c}\begin{array}{c}\text { Participación PIB } \\
\text { Agrop en el PIB } \\
\text { total }\end{array} \\
\% \text { Promedio } \\
1998-2000\end{array}$} & \multicolumn{2}{|c|}{$\begin{array}{l}\text { Gasto Público } \\
\text { Agropecuario como }\end{array}$} \\
\hline & & & & & $\begin{array}{c}\% \text { PIB } \\
\text { total }\end{array}$ & $\begin{array}{l}\text { \% PIB } \\
\text { Agrop }\end{array}$ \\
\hline $\begin{array}{l}\text { Mesoamérica } \\
\text { e ltsmo }\end{array}$ & 5677,00 & 507967,00 & 28272,00 & 5,57 & 1,12 & 20,08 \\
\hline México & 4621,00 & 450404,00 & 19499,00 & 4,33 & 1,03 & 23,70 \\
\hline Panamá & 139,00 & 9182,00 & 660,00 & 7,19 & 1,51 & 21,06 \\
\hline Honduras & 101,00 & 4476,00 & 850,00 & 18,99 & 2,26 & 11,88 \\
\hline Costa Rica & 105,00 & 14306,00 & 1692,00 & 11,83 & 0,73 & 6,21 \\
\hline El Salvador & 192,00 & 10757,00 & 1332,00 & 12,38 & 1,78 & 14,41 \\
\hline Guatemala & 432,00 & 16508,00 & 3412,00 & 20,67 & 2,62 & 12,66 \\
\hline Nicaragua & 87,00 & 2334,00 & 827,00 & 35,43 & 3,73 & 10,52 \\
\hline Caribe & 431,00 & 21160,00 & 2223,00 & 10,51 & 2,04 & 19,39 \\
\hline $\begin{array}{l}\text { República } \\
\text { Dominicana }\end{array}$ & 404,00 & 16020,00 & 1817,00 & 11,34 & 2,52 & 22,23 \\
\hline Jamaica & 27,00 & 5140,00 & 406,00 & 7,90 & 0,53 & 6,65 \\
\hline $\begin{array}{l}\text { Comunidad } \\
\text { Andina }\end{array}$ & 1262,00 & 256316,00 & 25465,00 & 9,94 & 0,49 & 4,96 \\
\hline Venezuela & 295,00 & 74384,00 & 3857,00 & 5,19 & 0,40 & 7,65 \\
\hline Ecuador & 188,00 & 18375,00 & 2212,00 & 12,04 & 1,02 & 8,50 \\
\hline Perú & 488,00 & 59372,00 & 5194,00 & 8,75 & 0,82 & 9,40 \\
\hline Colombia & 231,00 & 96380,00 & 13107,00 & 13,60 & 0,24 & 1,76 \\
\hline Bolivia & 60,00 & 7805,00 & 1095,00 & 14,03 & 0,77 & 5,48 \\
\hline Cono Sur & 7594,00 & 1106768,00 & 80430,00 & 7,27 & 0,69 & 9,44 \\
\hline Uruguay & 478,00 & 19929,00 & 1556,00 & 7,81 & 2,40 & 30,72 \\
\hline Paraguay & 91,00 & 8572,00 & 2227,00 & 25,98 & 1,06 & 4,09 \\
\hline Argentina & 1837,00 & 274825,00 & 13494,00 & 4,91 & 0,67 & 13,61 \\
\hline Chile & 848,00 & 85813,00 & 4768,00 & 5,56 & 0,99 & 17,79 \\
\hline Brasil & 4340,00 & 717629,00 & 58385,00 & 8,14 & 0,60 & 7,43 \\
\hline $\begin{array}{l}\text { Latinoamérica } \\
\text { y el Caribe }\end{array}$ & 14964,00 & 1892211,00 & 136390,00 & 7,21 & 0,79 & 10,97 \\
\hline
\end{tabular}

Fuente: estimaciones de Pensado, 2003. Estudio sobre el Gasto Público Agropecuario y de Áreas Rurales: 1985-2001, FAO. 


\section{Bibliografía}

Academia de Centroamérica; FAO; RUTA; Serfirural. Quirós, Rodolfo (editor). 2007. Financiamiento de las Cadenas Agrícolas de Valor.

Banco Mundial. 2006. Reducción de pobreza y crecimiento: círculos virtuosos y círculos viciosos.

BID. 2000. Informe anual sobre actividades de desarrollo rural.

BID. 2003. Informe rural.

CEPAL. Mayo 2004. Competitividad del sector agrícola y pobreza rural: el papel del gasto público en América Latina.

CEPAL/ICEFI. Julio 2006. Retos de la política fiscal en América Central.

CEPAL. Marzo 2005. Información básica del sector agropecuario: Subregión Norte de América Latina y el Caribe 1990-2003.

CEPAL. Mayo 2002. La banca multilateral de desarrollo en América Latina.

CEPAL. Junio 2005. Istmo Centroamericano: evolución del sector agropecuario 2003-2004.

CEPAL. Abril 2005. Los recursos hídricos y la agricultura en el Istmo Centroamericano.

IICA. 2006-2007. Perspectivas de la Agricultura y la Vida Rural en las Américas (primer borrador).

IICA. Septiembre 2006. Políticas para la Prosperidad Rural. Informe especial sobre la contribución de la agricultura y de los territorios rurales a la reducción de la pobreza, la creación de empleo y la promoción de la prosperidad rural.

IICA/De Miranda, Carlos Luiz (Supervisor). 2002. Brasil: Descentralización y Desarrollo Regional Sustentable. La Experiencia del Seridó.

IICA/C. Miranda y A. Matos. 2002. Desarrollo rural sostenible con enfoque territorial: La experiencia del IICA en Brasil. 
IICA/Espinoza, Nelson 2006. Política Agrícola Centroamericana (PACA). Grupo Técnico de Inversión Pública Agrícola. Análisis Preliminar de la Inversión Pública Agrícola del Istmo Centroamericano en el Contexto de América Latina y el Caribe.

IICA/Espinoza, Nelson. 2005. Las estrategias sectoriales: instrumentos necesarios para que los ministerios de agricultura trabajen más estrechamente con los de finanzas y otros actores claves (documento interno).

IICA-RUTA/Gtoro, NEspinoza. 2003. Los fondos competitivos para la agricultura y el desarrollo rural: fundamentos, aplicaciones y lecciones aprendidas.

IICA-RUTA/NEspinoza, JSmyle, JGatica. 2000. El pago de servicios ambientales y el desarrollo rural sostenible.

IICA/Medina, Héctor.2006. Perspectivas de la inversión y financiamiento en ALC y su relación con la agricultura (documento interno).

IICA/Miranda, Byron. 2006. Políticas Públicas y Dinámicas Territoriales en Centroamérica, presentación pp.

IICA/Sepúlveda, Sergio. 2002. Desarrollo Sostenible Microrregional. Métodos para Planificación Local.

IICA. Sepúlveda, Sergio y Rodriguez, Adrián. 2005. Sinopsis: Políticas para la Prosperidad Rural. № 5 .

www. worldbank.org. Projects and Operations.

www.iadb.org. Portal de proyectos. 


\section{ANEXO I \\ Análisis sobre el desarrollo fronterizo Costa Rica-Nicaragua}

\section{Comentario general}

Un primer comentario general con base en el análisis correspondiente de los documentos (ver listado al final), es que se observa un conjunto de numerosas iniciativas desarticuladas, desfasadas y duplicadas (en algunos casos, iniciativas que se están ejecutando son repetidas por nuevas propuestas). Obviamente, no ha habido forma de poder diseñar y poner en acción una figura institucional con liderazgo y con una carta de navegación clara que pueda dirigir el desarrollo de la región fronteriza. La sensación que se tiene, es que se han invertido importantes recursos y la situación de pobreza y creciente deterioro de los recursos naturales parece no haber cambiado mucho.

Un segundo comentario general, se refiere a la complejidad de coordinar acciones interfronterizas, considerando además, que hay diferencias notables en cuanto a indicadores socioeconómicos de desarrollo entre la población de la frontera de Costa Rica y la población de la frontera de Nicaragua.

Un tercer comentario general es la gran profusión de iniciativas de proyectos: (i) el Programa de Desarrollo Fronterizo propone una cartera de 28 proyectos con un costo estimado de US\$174 millones de los cuales los de infraestructura representan el $62 \%$ de los recursos, la parte productiva un $21 \%$, la medio ambiental un $6 \%$, la social un $8 \%$, y la gobernabilidad y fortalecimiento institucional un $3 \%$ (pocos recursos para el tema institucional y de gobernabilidad que requiere más apoyo); (ii) en cuanto a turismo rural, hay ocho proyectos regionales que tienen acciones en la zona fronteriza; hay cuatro proyectos en ejecución en Costa Rica y nueve proyectos nuevos que se han propuesto.

Un cuarto comentario general es que la atomización de iniciativas que se han impulsado y que se proponen para ambas fronteras; estas iniciativas adolecen de un gran proyecto aglutinador e integrador. Un proyecto bandera, que le de sentido de direccionalidad, rumbo o una carta de navegación a las iniciativas que se han ejecutado, que están en ejecución y que se están proponiendo para el futuro. 


\section{Comentarios especificos}

El análisis del Programa de Desarrollo Fronterizo Nicaragua-Costa Rica cuyo principal producto es una cartera de proyectos con costo total de US $\$ 174$ millones, adolece de varios defectos: (i) el programa debería haberse diseñado teniendo presente las iniciativas que están en ejecución o en negociación, de manera que se visualizara claramente el carácter complementario de las propuestas, o bien, el carácter innovador de las mismas, evitando duplicaciones; (ii) el programa debería haberse diseñado bajo la forma de un plan de negocios para que pueda respaldar las negociaciones con donantes y/o cofinanciadores de los proyectos en etapas iniciales de preinversión; (iii) respecto al costo total de cada proyecto, debería haberse hecho una diferenciación entre los costos de formulación y los costos de ejecución; esto tiene mucha utilidad en etapas tempranas de diseños de proyectos porque cuando se identifica a un donante $\mathrm{y} / \mathrm{o}$ cofinanciador es mucho más fácil comprometer los recursos de formulación que son montos mucho más pequeños que los de inversión, motivando o incentivando más el interés de los donantes y/o cofinanciadores en apoyar todo el proceso de preinversión e inversión.

El documento Trayectorias y Disyuntivas del Agro en la Zona Norte de Costa Rica, recopila ocho estudios y/o investigaciones, cuyas principales conclusiones son las siguientes: (i) existe una gran asimetría en el bienestar social de Nicaragua y Costa Rica; (ii) se estima que la tendencia, hacia el futuro, es que seguirá la deforestación acelerada, creando un gran impacto ambiental negativo, a lo cual seguirá contribuyendo la intensificación de la producción comercial empresarial agrícola dirigida a la exportación; (iii) tiene una importancia fundamental el tratamiento de la migración nicaragüense que viene a trabajar a esa zona, con políticas y acciones adecuadas que impidan la falta de respeto de los contratos y derechos sociales, que están provocando la disminución de los ingresos y desplazamiento de la mano de obra costarricense; (iv) ha habido un proceso de salarización de los pequeños productores y ha disminuido el trabajo familiar, porque se están empleando en el turismo y las empresas agroindustriales exportadoras (naranja, piña, banano, arroz, friijol, palmito, raíces, tubérculos, etc.); está en retirada la agricultura familiar campesina, que cumple con varias funciones: producción de bienes, generación de ingresos, protección de recursos naturales, paisajes rurales y mantenimiento de la cultura original; ( $v$ ) los servicios de transferencia de tecnología brindados por el MAG han disminuido y crecientemente lo están brindando las organizaciones locales, el Programa de Desarrollo Campesino, las empresas agroindustriales y universidades; ha habido experiencias exitosas en experimentaciones realizadas por los pequeños agricultores, su comunicación e intercambio entre ellos, pero con la terminación del Programa de Desarrollo Campesino esto ha ido decreciendo; 
(vi) la mayoría de las organizaciones campesinas enfrentan serios problemas de integración, comunicación y liderazgo por lo que hay que repensar las formas de apoyo a dichas organizaciones mediante mayor participación e integración; (vii) respecto a un análisis de la evolución de las acciones del ITCO/IDA, se concluye que hay descoordinación entre el discurso político y las políticas institucionales; y (vii) hay un estudio interesante sobre un proyecto apícola dirigido a la producción multidisciplinaria que está articulado con la reforestación y el manejo del bosque natural.

Del documento Síntesis sobre la Problemática de los Cantones Fronterizos de Costa Rica, se concluye que el modelo de desarrollo no ha producido equidad y acceso a los beneficios del desarrollo económico. Asimismo, se concluye que: existe sobreuso de suelos; la frontera agrícola se ha ampliado; falta la realización de un mapa sobre el uso actual y potencial del uso del suelo; ha disminuido el bosque y las cuencas hidrográficas han sido muy intervenidas con efectos negativos para el medio ambiente.

Del documento Diagnóstico Situacional de los Cantones Fronterizos, realizado por el INA y referido al tema de capacitación, se concluye que: la población de la región es joven, ya que, aproximadamente, el $51 \%$ tiene menos de 19 años; hay déficit en la educación secundaria y el acceso a la educación universitaria es muy limitado; la migración es negativa y hay una alta concentración de la tierra, ya que el $6 \%$ de los propietarios tiene el $65 \%$ de las tierras; falta una estrategia local para subsanar las deficiencias y hay que fortalecer la estructura dirigente para el desarrollo local (desarrollar el liderazgo); falta una visión estratégica a nivel binacional y una estrategia concertada entre las instituciones públicas y privadas.

Finalmente, del estudio Evaluación del Potencial del Turismo Rural de los cantones fronterizos de Costa Rica, se concluye que hay un enorme potencial para este tipo de iniciativas. Existen cuatro proyectos en ejecución, hay ocho proyectos regionales (centroamericanos) que tienen acciones en la zona y se proponen nueve proyectos nuevos.

\section{Conclusiones y recomendaciones}

Es evidente que cualquier iniciativa tendente a direccionar y poner orden en el desarrollo de la región fronteriza requiere de una figura institucional potente, con liderazgo y capacidad ejecutora. Por lo tanto, esa figura institucional pasa a ser una precondición indispensable para cualquier iniciativa. 
Para contribuir a consolidar la figura institucional propuesta, tiene que fundamentarse en una iniciativa potente, que aglutine intereses, que entusiasme y que permita articular, en función de dicha propuesta, el conjunto de iniciativas que existen y las que vendrán a futuro. Esa iniciativa podría ser alrededor de la cuenca del río San Juan, ya que es la unidad natural de funcionamiento de gran parte de las actividades socioeconómicas que existen en la región fronteriza. Además, alrededor de dicha cuenca se puede fundamentar con mayor fuerza todas las acciones necesarias para revertir el deterioro de la base de los recursos naturales y los impactos negativos en el ambiente; lo que incluiría, entre otras cosas, proyectos de protección y conservación, producción amigable con el ambiente, turismo (turismo rural, turismo de aventura, turismo científico, turismo de observación de flora y fauna, etc.). Además, el hecho de proponer algo positivo y constructivo hacia la cuenca, como por ejemplo, Salvemos a la Cuenca del río San Juan, Trabajemos Juntos por el Desarrollo Sostenible de la Cuenca del río San Juan, concita interés, apoyo y actitud positiva de parte de un amplio espectro de actores nacionales e internacionales.

Una de las primeras cosas que se tiene que hacer es poner orden en relación con todas las iniciativas que se están llevando a cabo y que se están negociando para el futuro; para ello, un primer paso debería ser un "mapeo" sistemático de los programas y proyectos, poniendo especial atención en sus alcances y resultados; la idea es que con un buen "mapeo" se puedan tener claros los temas y áreas donde hay iniciativas y donde no se está haciendo nada, para hacer un esfuerzo de complementación con las nuevas propuestas que han surgido y que están en negociación o que están engavetadas. También se requiere de un compromiso de cooperación y articulación por parte de los responsables y/o líderes que tienen a cargo los programas y proyectos.

Las iniciativas de proyectos articuladoras e integradoras o que vayan a cubrir temas o áreas que no se han planteado deben ser parte de un programa de inversión para la cuenca del río San Juan, que sea atractivo, flexible y muy vendible para los potenciales donantes y/o cofinanciadores. En este sentido, se podría plantear la figura de un plan de negocios en que se especifique tanto los requerimientos de recursos de preinversión para formular los proyectos a niveles más avanzados, así como, los costos de inversión para la ejecución de los proyectos. La idea es que en un etapa muy temprana del proceso de preinversión (con proyectos a nivel de idea o perfiles), y con un bajo costo, se logre interesar a los potenciales donantes y/o cofinanciadores, de manera que se puedan articular tempranamente en el proceso de preinversión-inversión. Debe diseñarse y poner en acción una estrategia de promoción y difusión muy bien pensada, apuntando a los actores claves para culminar con una gran reunión internacional de donantes. 
Listado de documentos analizados

Gobierno de Nicaragua y Gobierno de Costa Rica. 2005. Programa de Desarrollo Fronterizo. Costa Rica.

IICA. 2005. Síntesis sobre Problemática en los Cantones de Upala, Guatuso, Los Chiles y La Cruz. Costa Rica.

IICA-Costa Ricay Ministerio de Agriculturay Ganadería. Los Chiles, Upala, Guatuso y La Cruz: Mapas para un Desarrollo Rural Sostenible. Costa Rica.

Instituto Nacional de Aprendizaje. 2004. Diagnóstico Situacional de los Cantones de Guatuso, Upala y Los Chiles. Costa Rica.

PRODAR-IICA Costa Rica. 2005. Evaluación del Potencial del Turismo Rural en los Cantones de Intervención del Proyecto IICA-PDR (Upala, Los Chiles, Guatuso y La Cruz). Costa Rica

Universidad de Costa Rica y CIRAD. 2005. Trayectorias y Disyuntivas del Agro en la Zona Norte de Costa Rica. Costa Rica.

\section{ANEXO 2}

\section{La experiencia del IICA: lecciones aprendidas del desarrollo rural con enfoque territorial de Brasil}

El proceso de elaboración de planes y estrategias de desarrollo sostenible en los territorios rurales llevado a cabo con la cooperación técnica del IICA, posibilitó la construcción de un referencial técnico real, para las instituciones socias y para la comunidad en general. Durante toda la experiencia fue notoria la seriedad y la responsabilidad con que la sociedad respondió a las convocatorias de participación. Quedó ampliamente demostrada, además, su capacidad para reflexionar sobre su pasado, su presente y su futuro.

Es evidente que la población está interesada en decidir su destino, y cuando se le ofrece esa posibilidad se percibe que hay convicción sobre la veracidad de las informaciones intercambiadas entre los diferentes actores sociales. Como en todo proceso educativo, los resultados no necesariamente se perciben de forma inmediata; de ahí la importancia de insistir en hacer funcionar 
instrumentos y mecanismos de participación y ejercitar prácticas pedagógicas libertadoras.

Cabe señalar, sin embargo, que no existen ni recetas ni propuestas que aseguren el protagonismo de las comunidades en los procesos de planeación del desarrollo. La realidad será la encargada de definir los límites y las posibilidades de participación de los pobladores. Del mismo modo, la labor de planeamiento del desarrollo es un reto que debe superarse día a día, y debe contar con espacios de diálogo permanente, lo que significa motivar, en todo momento, la inclusión de actores sociales en la transformación que se pretende operar. A continuación ofrecemos una síntesis, organizada por temas, de las principales lecciones aprendidas:

\section{Aspectos conceptuales}

a) La experiencia adquirida por el IICA demostró, claramente, la necesidad de someter el marco conceptual, las metodologías y los instrumentos de planeamiento y gestión del desarrollo local sostenible a un proceso de perfeccionamiento constante. Todos esos elementos están sujetos a un ajuste permanente, puesto que se encuentran inmersos en la dinámica de las transformaciones del mundo rural y deben responder a la heterogeneidad y diversidad de los territorios rurales.

b) La aplicación del concepto de desarrollo sostenible implica la discusión de valores éticos y sociales, tales como la solidaridad intrageneracional e intergeneracional y el respeto a las leyes de la naturaleza. La implementación de un proceso de planeamiento llevará, sin duda, al debate de los valores dominantes en las comunidades y abrirá la posibilidad de cambiar aquéllos que no son compatibles con la sostenibilidad.

c) El concepto de desarrollo sostenible es abordado, en la planeación, desde una perspectiva multidimensional. Este ejercicio multidisciplinario incluye técnicos, especialistas y actores sociales variados, lo que permite calibrar cada una de las opciones en razón de las distintas dimensiones de la sostenibilidad.

d) Aproximarse a la realidad como un todo, con sus múltiples dimensiones, es una tarea compleja, pero posible si se utiliza el abordaje sistémico indicado en la metodología como un recurso de análisis y elaboración de propuestas.

e) La planeación del desarrollo sostenible exige el concurso de dos procesos, el 
técnico y el político, pues en todo momento se deben negociar las aspiraciones de la comunidad y las posibilidades económicas, tecnológicas y ambientales.

f) El resultado de la planeación y el grado de sostenibilidad del desarrollo están directamente influidos por la cultura y el momento político que vive la sociedad local.

g) La construcción de un proceso de planeación democrático, por su carácter legitimador de la acción pública, demostró ser más importante que el producto en sí, el plan. Esto rompe con una tradición reciente de planeación en el Brasil, en donde predomina la cultura del plan. En la práctica, esto significa vencer un desafío crucial: lograr que la planeación sea entendida como un proceso y no simplemente como un producto.

\section{Movilización de la sociedad}

a) La forma de propiciar la participación de la sociedad y la intensidad con que esto ocurre depende de la cultura y del momento políitico de cada territorio. Así mismo, con respecto a la sociedad en cuestión, no hay recetas generales para organizarla, dimensionarla, promoverla.

b) La participación real, efectiva, incluye el conjunto de los actores sociales y sus organizaciones; es un proceso complejo, demorado, que exige maduración. Su costo político es significativo, pero debe ser entendido como una inversión políica dirigida a la formación de la ciudadanía, del capital social, del empoderamiento de los actores sociales y de la legitimidad de las acciones públicas futuras.

c) Para que tengan permanencia en el tiempo, las acciones de desarrollo local sostenible referidas a la movilización de la sociedad, a la organización de los actores sociales y a la reacción de instancias deliberativa deben implementarse en el contexto de proyectos de inversión, siguiendo el ejemplo de los Proyectos de Combate a la Pobreza Rural (PCPR).

d) La legitimidad de los representantes de cualquier segmento social merece un cuidadoso análisis previo, porque ellos reflejan la estructura de poder y los conflictos de intereses de cada territorio. Por eso, la metodología utilizada por el IICA comienza exactamente con esta evaluación. 
e) El esfuerzo de movilización y organización comunitaria debe estar siempre atado a proyectos de inversión, pues, de esta forma, es posible asociar la mejoría de la calidad de vida al ejercicio de la ciudadanía.

f) Las dificultades de movilización son muy comunes en este proceso; por ello, este aspecto merece un tratamiento especial permanente. El establecimiento de estrategias y amplios sistemas de alianzas para reducir esas dificultades, como en el caso del Seridó, en el que participaron iglesias, movimientos sociales y poderes públicos, resulta fundamental.

\section{Capital social}

a) Cuanto más fuertes sean las organizaciones de base, mayor será el éxito del proceso de planeación y de gestión de planes de desarrollo sostenible. La creación de instancias deliberativas formales (consejos, foros) es necesaria, pero no suficiente. Cuanto mayor sea la incorporación de las organizaciones de base y su representación a las instancias deliberativas, mayor será la posibilidad de que éstas permanezcan en el tiempo y mejores los resultados alcanzados.

b) La calidad y la legitimidad de los liderazgos no está directamente relacionada con el mandato político. La fragilidad del liderazgo, con o sin mandato, compromete la sostenibilidad política de la propuesta.

c) Desde un inicio es preciso invertir esfuerzos en la identificación de los patrocinadores del proceso, en la sensibilización de los actores que van a participar y en el diseño de mecanismos de participación. Es necesario, además, demostrar que la elaboración del plan es apenas un momento de un proceso más amplio, el cual debe continuar bajo el patrocinio de quienes tienen legítima capacidad aglutinadora para conducirlo y para formar parte de la nueva institucionalidad que exige el desarrollo local sostenible

d) Las redes de cooperación y solidaridad social deben ser valorizadas y promovidas, pues consideran la fortaleza o la fragilidad de sus estructuras o del trabajo que individualmente realizan.

\section{Capital humano}

a) A lo largo de su experiencia, el IICA observó que para trabajar con desarrollo local sostenible es necesario contar con profesionales capaces de: 
i. Situar el territorio que se está trabajando en el contexto regional, nacional y mundial.

ii. Dominar técnicas de planeación y gestión, y técnicas de relación con distintos actores sociales.

iii. Trabajar con cadenas de agronegocios.

iv. Respetar y valorar las diferencias.

v. Reconocer aquellos valores locales que posean ventajas comparativas con respecto a distintos mercados.

vi. Mostrar disposición a intercambiar saberes.

b) En todas las experiencias del IICA, la utilización de metodologías participativas de planeación condujo a una mayor motivación y autoestima de los profesionales del sector público participantes.

\section{Territorio}

a) La delimitación holística, sistémica e integrada del concepto de territorio sostenible, posibilita:

i. Ganancia de economías de escala, modernización de los procesos productivos y mayor gobernabilidad de los factores ambientales.

ii. Mayor respeto a las especificidades culturales.

iii. Mejor aprovechamiento de las potencialidades locales, lo que significa explorar las ventajas comparativas agrícolas o no agrícolas de cada territorio.

iv. Participación más efectiva de los actores sociales en la planeación, en la gestión y en el control social de las políticas públicas.

v. Mayor dinamismo de los procesos de organización de los actores sociales.

vi. Establecimiento de un pacto político entre el sector público y las entidades civiles. 
vii. Compatibilización de los procesos técnico y políticos, con el propósito de integrar las propuestas de la sociedad en las respuestas técnicas, a partir de un ambiente de negociación permitido a todo lo largo del proceso de elaboración del plan.

viii. Mejor organización y capacitación de los equipos técnicos multidisciplinarios e interinstitucionales.

b) El concepto de territorio se vale de otros aspectos además de los meramente técnicos y político-administrativos. La cultura local es un elemento fundamental en la delimitación de los territorios rurales y se iguala a otras variables como las sociales, las geográficas, las demográficas y las económicas. Un ejemplo esclarecedor lo ofrecen los territorios quilombolas del Maranhão, constituidos por 42 comunidades, esparcidas en 8 municipios, que aun cuando no ocupan un territorio continuo, están integrados en razón de su cultura y su etnia.

c) La organización social y el capital social son elementos fundamentales para la conceptualización y delimitación de un territorio. Hoy día, por ejemplo, los habitantes del Seridó reconocen su territorio no solo por sus características climáticas, geográficas y demográficas, sino por las 450 asociaciones comunitarias, 25 cooperativas, 26 sindicatos rurales, 28 consejos municipales, 2 asociaciones de municipios y un consejo de desarrollo regional que lo conforman. Además, ven en él las redes de cooperación y confianza que se han establecido entre las distintas asociaciones.

d) Cuanto más se reduce la escala geográfica del territorio más, fácil se torna combinar el proceso técnico y el político. Lo inverso también es cierto, mas hay que considerar que las escalas menores perjudican la concepción y ejecución de programas estructurantes.

e) El análisis del entorno nacional e internacional del territorio probó ser de gran importancia para la identificación de las amenazas y las oportunidades que inciden en él.

\section{Estrategias de intervención}

a) La realidad social es "per se" conflictiva; de ahí que se recomienda explicitar y tratar con transparencia las diferencias, no desconocerlas. En la experiencia del IICA, los conflictos surgieron en todas las etapas de formulación de los 
planes, e incluso se agudizaron durante la formulación de las estrategias de intervención, por ser este el momento en que los intereses de los distintos actores sociales y las diferencias entre el sector público y el privado emergen con más claridad.

b) El planeamiento es un proceso político de toma de decisiones y de negociaciones entre diversos actores. El plan es, ante todo, un instrumento de negociación y de aglutinación política, en la medida en que expresa, de forma técnica y organizada, el conjunto de las decisiones y los compromisos asumidos por los que participan en el proceso de su construcción o por quienes están dispuestos a ejecutarlo.

c) El hecho de que las entidades organizacionales de los diversos niveles de gobierno estén estructuradas por sectores, visión que de cierta forma se reproduce en las representaciones de la sociedad civil, acarrea una dificultad metodológica que merece especial consideración a lo largo del proceso. Organizar la discusión por dimensión implica romper con una postura tradicional, y esto exige una motivación adicional para que las personas "abandonen" sus sectores, y pasen a hacer análisis y propuestas con una visión holística de la realidad. Eso no siempre es posible.

d) La necesidad, causada muchas veces por el alto grado de pobreza del territorio, de buscar resultados económicos de corto plazo, puede generar impactos indeseables en el medio ambiente. Tales impactos deben ser estimados y analizados cuidadosamente para orientar las decisiones en el ámbito económico. En el sentido contrario, los procesos de alteración del medio ambiente que maduran lentamente pueden tener implicaciones en el mediano y largo plazos sobre el desempeño de la economía.

e) La discusión de las diversas dimensiones, simultáneamente, en momentos de síntesis, fue el medio que se adoptó para destacar e identificar soluciones apropiadas provocadas por choque de objetivos.

f) La gestión pública local toma en consideración las relaciones entre el poder público local y las organizaciones no públicas prestadoras de servicios sociales. La Prefectura jamás podría oponerse al plan, por razones muy simples: El Brasil posee cerca de 5.700 municipios, de los cuales un $76 \%$ tiene menos de 20 mil habitantes y un $90 \%$ de menos de 50 mil habitantes. En este conjunto que constituyen los más pobres, la Prefectura es la principal empresa, puesto que emplea el mayor número de personas y es responsable por la mayoría de 
los servicios que se prestan a la población, como educación y salud, además de recibir las transferencias del gobierno federal y estatal. Por otra parte, hay una evidente correlación positiva entre los niveles de Índice de Desarrollo Humano (IDH) y el desempeño emprendedor de las prefecturas. Esta situación justifica la implementación de programas complementarios que contribuyan al fortalecimiento institucional de las prefecturas.

g) La generación de empleo y renta considera las actividades agrícolas y las no agrícolas para garantizar la sostenibilidad económica del desarrollo de los territorios rurales, por medio de la diversificación de sus economías. Las cadenas de agronegocios son vistas como una estrategia de generación de empleo, renta y fortalecimiento del capital social.

h) En razón del nivel de pobreza existente, se debe combinar el plan de acciones estructurantes (crédito y reforma agraria), con una serie de acciones compensatorias inteligentes (financiamiento de sistema simplificado de abastecimiento de agua con gestión comunitaria, por ejemplo). Cabe señalar, sin embargo, que con estas últimas, se corre el riesgo de volver a caer en el asistencialismo, al que se sienten muy apegadas las regiones pobres.

i) La complementación de acciones macro con acciones micro es fundamental para la sostenibilidad del desarrollo. La existencia de millares de pequeños proyectos comunitarios en el Seridó no confirió la escala para la sostenibilidad del desarrollo de la región, y sirvió apenas para resolver problemas puntuales. Este esfuerzo deberá complementarse con la ejecución de programas de inversión de ámbito regional, como los programas de recursos hídricos y de cadenas de agronegocios previstos en el plan de desarrollo de la región. 